\title{
Свойства полупроводниковых коллоидных квантовых точек, полученных в условиях управляемого синтеза
}

\author{
(ㄱ Н.Д. Жуков ${ }^{1}$, Т.Д. Смирнова ${ }^{2}$, А.А. Хазанов ${ }^{1}$, О.Ю. Цветкова ${ }^{1}$, С.Н. Штыков ${ }^{2}$ \\ ${ }^{1}$ Общество с ограниченной ответственностью „НПП Волга“, \\ 410033 Саратов, Россия \\ ${ }^{2}$ Саратовский государственный университет им. Н.Г. Чернышевского, \\ 410012 Саратов, Россия \\ E-mail: ndzhukov@rambler.ru
}

Поступила в Редакцию 29 июня 2021 г.

В окончательной редакции 20 июля 2021 г.

Принята к публикации 2 августа 2021 г.

\begin{abstract}
Исследовано влияние технологических параметров синтеза на структурно-физические свойства коллоидных квантовых точек на основе сульфида свинца, антимонида индия и селенида кадмия. Использование несольватирующего для прекурсора неметалла растворителя позволяет получить медленный, управляемый процесс и детально проследить все стадии кристаллизации наночастиц - нуклеацию и рост, оствальдовское созревание, неконтролируемое разрастание. Удается получать кристаллиты совершенной структуры и размеров с минимальными разбросами - не более $\pm 10 \%$. Установлено при этом, что максимальные размеры наночастиц ограничены термодинамическими условиями роста и имеют величину $\sim 5$ нм. Кроме того, квантовые точки проявляют ряд специфических свойств - аномальную температурную зависимость фотолюминесценции и нестабильность вольт-амперной характеристики, которым дано объяснение в модели размерного квантования энергии и импульса неравновесных электронов. Для разных вариантов полупроводников особенности проявляются значительней с увеличением параметров размерного квантования.
\end{abstract}

Ключевые слова: коллоидный синтез, коллоидная квантовая точка, кинетическая и термодинамическая модели, сольватирующий растворитель, быстрая и медленная кристаллизация, кристаллическая и зонная структура, параметры размерного квантования, фотолюминесцентные и вольт-амперные характеристики.

DOI: 10.21883/FTP.2021.12.51706.9704

\section{1. Введение}

Одним из быстро развиваемых направлений полупроводниковой научной и практической проблематики является синтез, исследование свойств и возможностей применений коллоидных квантовых точек (QD) [1-3]. При этом доминирующей тенденцией исследований являются управляемый коллоидный синтез и свойства наночастиц разных форм: одномерных - нитей, двумерных - пластинок, трехмерных - полигональных и шарообразных QD [4,5]. Основная практическая направленность этих работ связана с опто- и наноэлектроникой $[6,7]$.

Форма и размеры коллоидных квантовых точек, их кристаллическая структура влияют на их физические свойства и возможности применений. Электронные процессы и квантовые состояния в QD обусловлены резонансным движением электронов в условиях квантоворазмерного ограничения [2,7]. Этот процесс зависит от структуры зон, энергетической и Бриллюэна, которые в свою очередь определяются степенью совершенства кристаллической решетки QD $[7,8]$.

Несмотря на большое число работ и публикаций, главная проблема технологии коллоидного синтеза квантовых точек - получение их нужной формы и узкого разброса по размерам - остается достаточно сложной. Технологические проблемы, как правило, решаются под конкретные условия исследований и при- менений [2,3]. При этом определяющим технологическим параметром при синтезе является степень пересыщения раствора, выражаемая на практике в скорости кристаллизации. В технологиях полупроводниковых структур скорость роста кристаллической структуры - определяющий интегральный параметр для любого процесса образования структуры кристаллической фазы. В коллоидной химии наночастиц интегральным параметром процесса является его время, варьируемое на практике от единиц секунд до десятков минут.

Цель нашей работы - установление определенных закономерностей коллоидного синтеза квантовых точек на основе сравнительного исследования структурнофизических свойств наиболее изучаемых вариантов, в основном сульфида свинца, полученных при технологическом варьировании состава растворителя, температуры, соотношения концентраций прекурсоров, времени процесса.

\section{2. Методика исследования}

Коллоидный синтез квантовых точек QD-PbS проводили с учетом данных методики, описанной в работе [9], в среде безводного уайт-спирита или октадецена с использованием в качестве прекурсоров олеатов свинца, серы и сероводорода. При этом использование нескольких 
типов растворителей, значительно отличающихся предельной растворимостью серы, нескольких источников прекурсора и разной температуры позволило получить зависимость размера и формы кристаллов QD от указанных параметров. Коллоидный синтез квантовых точек $\mathrm{InSb}$ проводили по методике, апробированной ранее [10], в среде безводного олеиламина, с использованием в качестве прекурсоров смеси ацетата и хлорида индия в соотношении 4:1 и трис[бис(триметилсилил)амида] сурьмы $\mathrm{Sb}\left[\mathrm{N}\left(\mathrm{Si}-(\mathrm{Me})_{3}\right)_{2}\right]_{3}$. Квантовые точки на основе $\mathrm{CdSe}$ изготовлены по технологии, описанной в работе [11].

Образцы квантовых точек синтезированы в лабораториях НПП „Волга“ (сульфид свинца), НИИ ПА Дубна (сульфид свинца-кадмия, селенид-сульфид кадмия, антимонид индия), Института химии Саратовского государственного университета (селенид-сульфид кадмияцинка).

Исследование основано на результатах измерений методами электронной (сканирующей - СЭМ, просвечивающей - TЕМ) и сканирующей зондовой (туннельной - СТМ, атомно-силовой - АCM) микроскопии, а также вольт-амперных характеристик (BAX) и фотолюминесценции (ФЛ). Использование методов электронной и сканирующей зондовой микроскопии для исследования свойств кристаллических полупроводников, в частности квантовых точек, описано, например, в работах $[12,13]$.

Использованные в нашей работе методы исследования QD позволяли контролировать следующие структурнофизические свойства: СЭМ - элементный состав наночастиц; ТЕМ - плоскую форму и размеры кристаллитов; СТМ - вольт-амперные характеристики отдельных наночастиц в планарной наноструктуре, выращенной по методу Ленгмюра-Блоджетт; ФЛ — фотолюминесцентную способность ансамбля наночастиц.

Элементный состав и стехиометрию наночастиц контролировали рентгеновским микроанализом на сканирующем электронном микроскопе MiraII LMU, оснащенном системой энергодисперсионных спектрометров INCAEnergy 350 (TESCAN, Чехия). Экспериментальная погрешность определения атомного состава материала была не хуже одного процента. Непосредственно перед измерениями квантовые точки освобождали от сорбированных на их поверхности лигандов и антиоксиданта отделением осадка центрифугированием (1 мин $10000 \mathrm{~g})$, редиспергировали в гексане и высаживали островками на кремниевую подложку толстым слоем (не менее 10 мкм). Для исследований использовались только партии образцов после контроля состава QD.

Форму и размеры квантовых точек исследовали на ТЭМ, с разрешением не хуже $0.3 \mathrm{нм}$, по общепринятой методологии [8]. Контроль размеров и формы QD позволяет качественно судить об их кристаллической структуре с учетом того, что закономерный рост кристаллита определяет полигональный характер и параметры его геометрической фигуры. Исследования проводили на просвечивающем микроскопе Libra-120 (CarlZeiss, Германия). Измерения размеров наночастиц проводили по ТЕМ-снимкам, при большом увеличении на экране, простым счетом рядами по 20-30 частиц подряд, общим количеством на каждом образце - не менее 100.

СТМ-исследование ВАХ позволяет определить механизмы электронного транспорта в QD. Измерения проводили по методам, описанным в работах [14-16]. Результаты измерений обрабатывали по общепринятым методикам сканирующей зондовой микроскопии полупроводниковых наноструктур [17].

ФЛ-исследование позволяет судить о механизмах возбуждения и рекомбинации неравновесных носителей заряда в QD и влиянии на них размерного квантования. Измерения проводились методом, описанным в нашей работе [18].

Все измерения проводили на случайных выборках, используя аликвоты коллоидного раствора изготовленных образцов.

Измерения на электронных микроскопах проводили в лабораториях ИБФРМ РАН (Саратов, ТЭМ) и Института наноструктур и биосистем СГУ (СЭМ).

\section{3. Результаты и обсуждение}

В работе исследованы следующие варианты квантовых точек (в скобках указаны химические формулы оболочки): $\mathrm{PbS}, \mathrm{PbS}(\mathrm{CdS})$, InSb-big, InSb-smal, InSb(InP/CdS), $\mathrm{CdSe}(\mathrm{CdS}), \mathrm{CdSe}(\mathrm{CdS} / \mathrm{ZnS})$.

Данные по параметрам синтеза квантовых точек приведены в таблице, где номерами 1-8 обозначены образцы QD-PbS, 9 - QD-PbS(CdS), 10 - QD-InSb-big, 11 QD-InSb-smal, 12 - QD-CdSe $(\mathrm{CdS} / \mathrm{ZnS})$. Данные приведены для отдельных выбранных из партий, как типичные, образцов. Было проведено более 30 экспериментов, по каждой вариации - по 2-3 эксперимента.

Сравнительный анализ о влиянии на время процесса и размеры QD проводился по следующим основным технологическим параметрам: по составу растворителя октадацен, уайт-спирит, олеиламин, ксилол; по температуре процесса $-20,150,200,250^{\circ} \mathrm{C}$; по соотношениям концентраций первого (свинец, индий) и второго (сера, сурьма) прекурсоров - образцы 1-4, 7-9, 10-11.

Основные выводы, следуемые из анализа данных таблицы, сводятся к тому, что коллоидный синтез в нашем случае относительно малочувствителен к концентрациям прекурсоров и температуре процесса, но резко зависит от типа растворителя, основным свойством которого является степень растворимости прекурсора и перевода его в активное состояние (сольватация). При этом главным критерием является скорость кристаллизации, выраженная временем процесса, а он сам может быть быстрым - не управляемым, и медленным управляемым.

Увеличение температуры раствора от 423 до $473 \mathrm{~K}$ для управляемого синтеза (образцы 7 и 8) приводило к ускорению процесса по времени в $\sim 4$ раза. Принимая, что кинетические свойства процесса, в зависимости от 
Данные по параметрам синтеза и размерам квантовых точек

\begin{tabular}{|c|c|c|c|c|c|c|}
\hline $\begin{array}{l}\text { № } \\
\text { П.П. }\end{array}$ & Растворитель & $\begin{array}{c}\text { Температура, } \\
{ }^{\circ} \mathrm{C}\end{array}$ & $\begin{array}{c}\text { Концентрация } \\
\text { первого } \\
\text { прекурсора, М }\end{array}$ & $\begin{array}{c}\text { Концентрация } \\
\text { второго } \\
\text { прекурсора, М }\end{array}$ & $\begin{array}{c}\text { Время роста, } \\
\text { мин }\end{array}$ & $\begin{array}{c}\text { Размеры QD, } \\
\text { нм }\end{array}$ \\
\hline 1 & Октадецен & 200 & 0.01 & 0.01 & $\sim 0.5$ & $7 \pm 3$ \\
\hline 2 & Октадецен & 200 & 0.15 & 0.15 & $\sim 0.2$ & $8 \pm 4$ \\
\hline 3 & Уайт-спирит & 200 & 0.15 & 0.01 & 3 & $2.5 \pm 0.5$ \\
\hline 4 & Уайт-спирит & 200 & 0.01 & 0.01 & 5 & $3.0 \pm 1.0$ \\
\hline 5 & Ксилол & 150 & 0.1 & $\mathrm{H}_{2} \mathrm{~S}$ & $\sim 0.2$ & $4 \pm 1.0$ \\
\hline 6 & Уайт-спирит & 20 & 0.15 & $\mathrm{H}_{2} \mathrm{~S}$ & $\sim 0.5$ & $4.5 \pm 1.0$ \\
\hline 7 & Уайт-спирит & 200 & 0.1 & 0.05 & 10 & $3.2 \pm 0.5$ \\
\hline 8 & Уайт-спирит & 150 & 0.1 & 0.05 & 40 & $4.5 \pm 0.5$ \\
\hline 9 & Олеиламин & 150 & 0.1 & 0.1 & $\sim 0.5$ & $4.5 \pm 0.5$ \\
\hline 10 & Олеиламин & 250 & 0.2 & 0.1 & 10 & $12 \pm 1$ \\
\hline 11 & Олеиламин & 250 & 0.2 & 0.1 & 10 & $7 \pm 0.5$ \\
\hline 12 & Октадецен & 140 & 0.1 & 0.1 & 10 & $7.5 \pm 0.5$ \\
\hline
\end{tabular}

температуры $T$, определяются формулой Арениуса $\exp (\breve{E} / k T)$, где $\breve{E}$ - энергия активации процесса, $k-$ постоянная Больцмана, и проводя вычисления для случая четырехкратного роста скорости, с расчетом на одинаковые значения размера QD, получили для параметра $\breve{E} \sim 0.1-0.3$ эВ.

Коллоидный синтез квантовых точек лимитируется по крайней мере двумя процессами - движением молекул и частиц в растворе и вероятностью их встречи (кинетическая модель); реакцией соединения и образования кристаллита (термодинамическая модель). Судя по полученным результатам, в рассматриваемом случае определяющей можно считать кинетику процесса. На это указывают такие полученные данные, как низкое значение энергии активации процесса, характерное для броуновского движения частиц в растворе, и сильная зависимость скорости синтеза от свойств растворителя, лимитирующих процесс перевода прекурсора в его активное состояние в растворе.

Измерения структурно-физических свойств QD проводили многократно, используя в каждой партии несколько образцов. На рис. 1, $a$ представлены типичные картины снимков фрагментов групп квантовых точек нескольких типов. Статистический анализ показал, что плоская форма квантовых точек имеет полигональный вид. При этом преимущественно для QD-PbS и CdSe преобладает четырех-пятигранная форма, a для QD-InSb и в отдельных случаях вариантов QD-PbS-трех-четырехгранная. Эти результаты указывают на то, что синтезированные кристаллиты имеют структуру, соответствующую сингонии кристаллической решетки полупроводников [19], а именно $\mathrm{CdSe}$ - гексагональную, $\mathrm{PbS}$ - гексагональную или кубическую, InSb - кубическую. Кроме того, исследование трехмерной формы по 3D-топограммам АCM позволило установить наличие уплощенной формы наночастиц, что может быть связано с тем, что при синтезе быстрее происходит рост кристаллита в направлении минимума энергии взаимодействия (связи). Для суль- фида свинца, например, энергетическая анизотропность решетки сравнительно велика и имеет минимум энергии связи в плоскости (100) [20].

Форма и размеры синтезированных QD во всех случаях и вариантах имели больший или меньший разброс. При этом для медленного процесса удается получить наилучшие результаты - выраженную полигональную форму кристаллитов и медианный разброс по размерам, не хуже $\pm 10 \%$.

Используя несольватирующие растворители, например уайт-спирит для серы, оказалось возможным проследить модельную кинетику процесса коллоидного синтеза. Рис. $1, b$ демонстрирует последовательность изменений для образцов QD-PbS типа 3-4 (см. таблицу) при увеличении времени процесса: стадия 1 ( 2мин) нуклеация и рост, характеризуемые большим разбросом размеров и формы кристаллитов (рис. 2, кривая 1); стадия 2 ( 4 мин) - освальдовское созревание, характеризуемое растворением частиц меньших размеров (с большой поверхностной энергией) и сужением разброса размеров (рис. 2, кривая 2); стадия 3 $(\sim 5-6$ мин $)$ - неконтролируемое разрастание наночастиц с увеличением разброса размеров (рис. 2, кривая 3) и переходом преимущественно к круглой форме наночастиц.

Полученные нами ТЕМ-изображения, форма и разбросы размеров QD качественно сходны с известными из литературы, например, [21,22] (рис. 1,c).

В целом модель роста может быть представлена следующим образом: зародыши правильной кристаллографической формы с размерами $<2$ нм и количеством атомов $<50$ развиваются закономерным ростом в несколько искаженные по форме кубики с размерами до $\sim 4$ нм и количеством атомов до 500, которые затем превращаются в многогранники, несколько искаженной формы с размерами до 6 нм и количеством атомов до 2000. Последующий (неконтролируемый) рост приводит к шароподобным фигурам больших наноразмеров. 


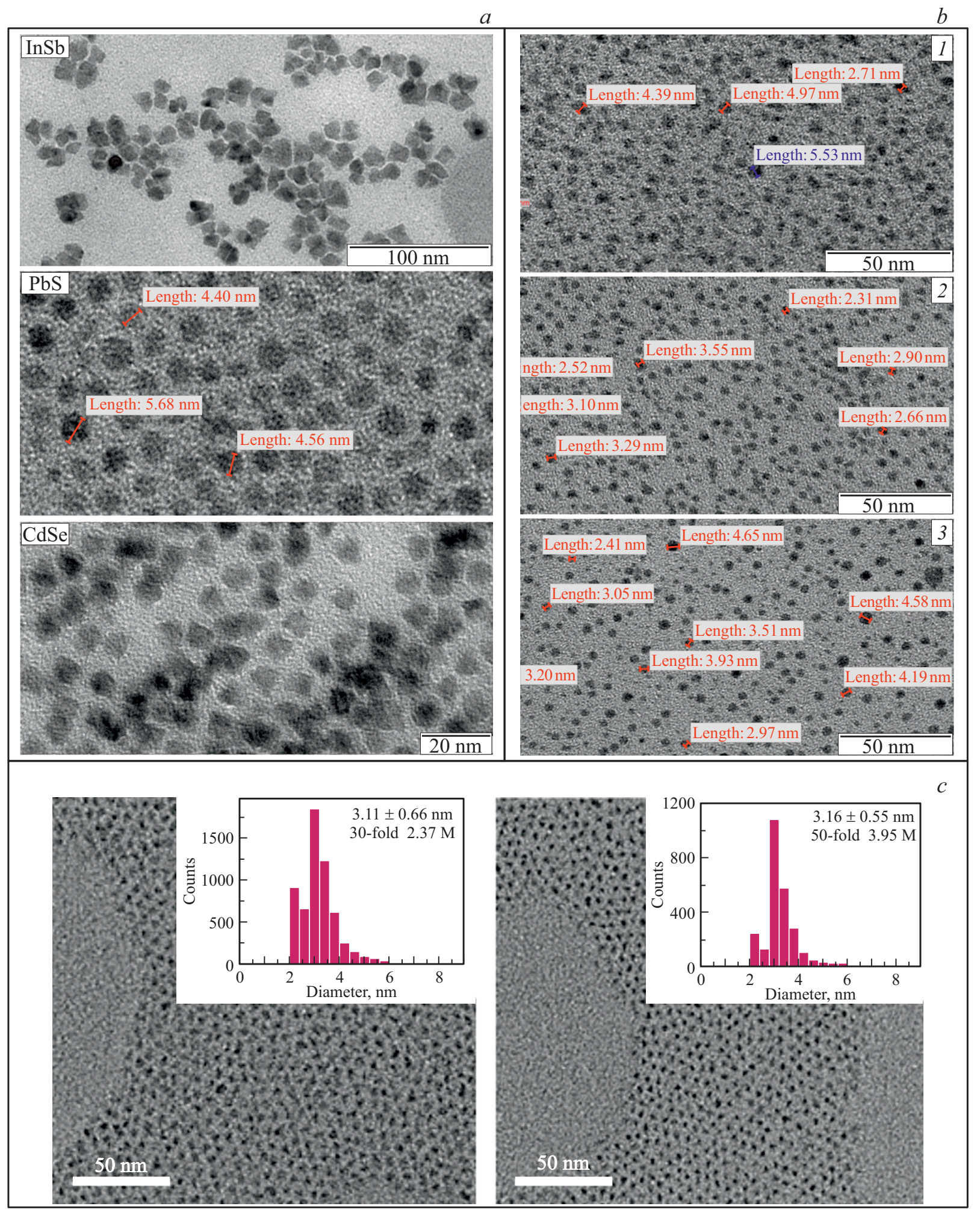

Рис. 1. Фрагменты ТЕМ-снимков квантовых точек: $a$ - основные варианты квантовых точек, синтезированных при управляемом росте; $b-$ фрагментарные ТЕМ-снимки для трех стадий синтеза: 1 - нуклеация и рост; 2 - оствальдовское созревание; 3 - неуправляемый рост; $c$ - качественно схожие снимки, как пример, из работы [23].

Рост кристаллитов QD связан с увеличением энергии и уменьшением энтропии его термодинамической системы. Начиная с некоторого размера, система перестает быть чувствительной к прибавляемым элементам - они могут быть чужими атомами и молекулами, скоплениями своих атомов, наночастицами. Кристаллы начинают 


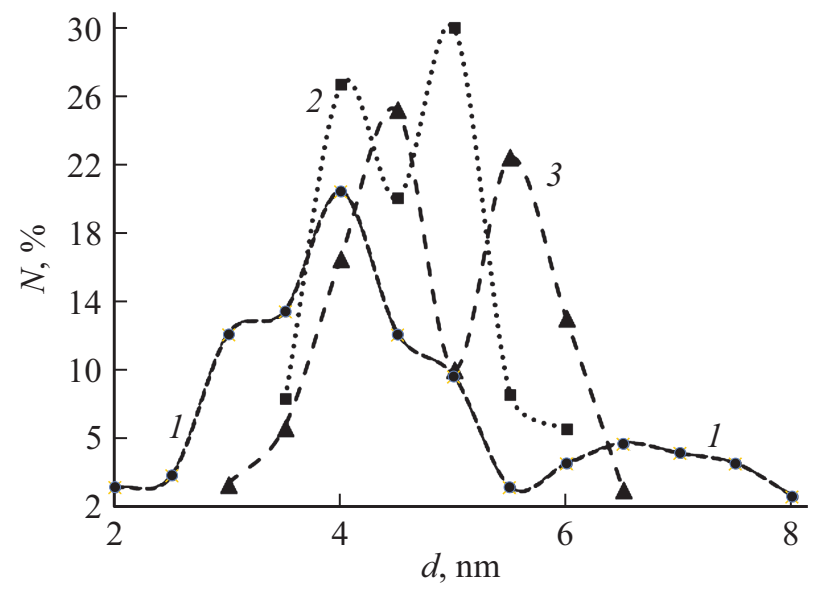

Рис. 2. Гистограммы распределения количества наночастиц по размерам для трех стадий синтеза: 1 - нуклеация и рост; 2 - оствальдовское созревание; 3 - неуправляемый рост.

расти за счет присоединения элементов силой Вандер-Ваальса с изотропным развитием - сферической формы.

При росте кристалла, когда у него появляется объемная форма и, естественно, поверхность, он будет расти эпитаксиально. По теории эпитаксии, на его поверхности образуются зародыши из небольшого числа атомов, которые, мигрируют по поверхности, пока не сомкнутся, наращивая кристалл и увеличивая энергию его термодинамической системы на величину $E_{\text {con. }}$. Отношение этой энергии к энергии связи атомов в кристалле, которая, видимо, близка к энергии образования кристалла $H_{e d u}$, предположительно, может быть примерно равно отношению постоянной решетки $a$ к размеру кристалла $d-E_{\text {con. }} / H_{e d u} \sim a / d$. Откуда $d \sim a\left(H_{e d u} / E_{\text {con. }}\right)$. Для

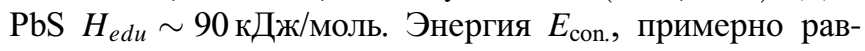
ная энергии Ван-дер-Ваальса, $-\sim 10$ кДж/моль. Тогда $d \sim 9 a \sim 4-5$ нм. Этот результат может свидетельствовать о том, что кристалл QD совершенной структуры может иметь размеры не более 5 нм. С другой стороны, QD больших размеров будут иметь шаровидную форму. Этот вывод подтверждается рис. 1.

Физические свойства QD зависят от движения электрона, описываемого квантово-механическим уравнением Шредингера. Математический формализм решения уравнения Шредингера позволяет сформулировать ряд важных электронных свойств в квантовой материальной среде, занимающей ограниченную область пространства [23]. Решение уравнения Шредингера существует только для счетного множества значений энергии $\tilde{E}_{n}$ и представляет собой счетное квантовыми числами $n$ множество волновых функций $\Psi_{n}$, создающих общее решение как аддитивную суперпозицию элементов множества. Для одномерного движения электрона волновая функция $\Psi_{n}$ дискретного спектра $\tilde{E}_{n}$ обращается в нуль (при конечных значениях координаты $x$ ) $n$ раз (осцилляционная теорема). Эти свойства позволяют упростить задачу, сведя ее к частному одномерному линейному случаю дистанции $x_{0}$ и прямоугольного потенциала $V_{0}$. Тогда уравнение Шредингера примет легко решаемую форму, а его частное решение относительно $\Psi_{n}$ и собственной функции $E_{n}$ выглядит следующим образом: $\Psi_{n}=8 m V_{0}\left(h^{2} n^{2}\right)^{-1} \sin \left(n x / x_{0}\right), E_{n} \sim h^{2} n^{2}\left(8 m x_{0}^{2}\right)^{-1}$. В рассматриваемой квантовой протяженной яме происходит отбор устойчивых состояний электрона так, чтобы на линейной дистанции его движения укладывалось целое число полуволн де Бройля. Всякие энергетические воздействия на QD, например, полем и светом, приводят к выводу из одного устойчивого состояния в другое. Устойчивость состояния движущегося неравновесного электрона в глубокой потенциальной яме и ее стоячая волновая функция могут означать, что это движение имеет резонансный характер. В этом случае размерное квантование энергии и импульса электронов в QD должно привести к неким особенностям их свойств, основные из которых, как показал анализ характеристик, сводятся к аномальной температурной зависимости уровня фотолюминесценции (рис. 3) и резкой смене механизма электронного транспорта (рис. 4) при некоторых определенных значениях напряжения на QD.

B общепринятой экситонной модели фотолюминесценции ее температурная зависимость определяется зависимой от температуры величиной эффективной массы экситона, которая с ростом температуры увеличивается. Это ведет к уменьшению энергии связи экситона и, соответственно, к уменьшению вероятности его состояния и времени жизни. В связи с этим с ростом температуры относительная интенсивность ФЛ QD-CdSe линейно уменьшается (рис. 3, кривая 2).

Таким же образом можно объяснить ветвь спада фотолюминесценции с ростом температуры для QD-PbS (рис. 3, кривая 1), но нельзя объяснить ветвь спада в сторону меньших температур. Объяснение этому явлению можно дать, учитывая, что в квантовых точках с высокими параметрами размерного квантования, какими являются QD-PbS, происходит конкуренция эффектов, когда квантово-размерное ограничение, приводящее к состоянию резонансного движения электрона, уменьша-

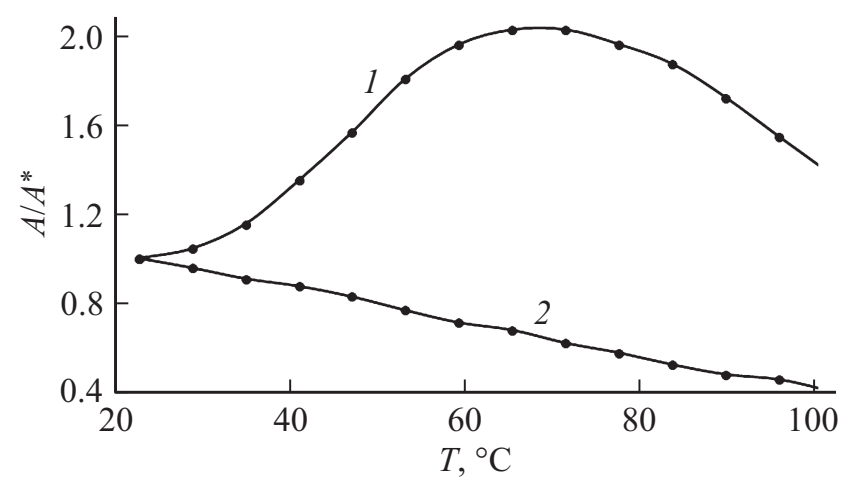

Рис. 3. Интегральные зависимости фотолюминесценции: 1 - QD-PbS(CdS); 2 - QD-CdSe(CdS). 


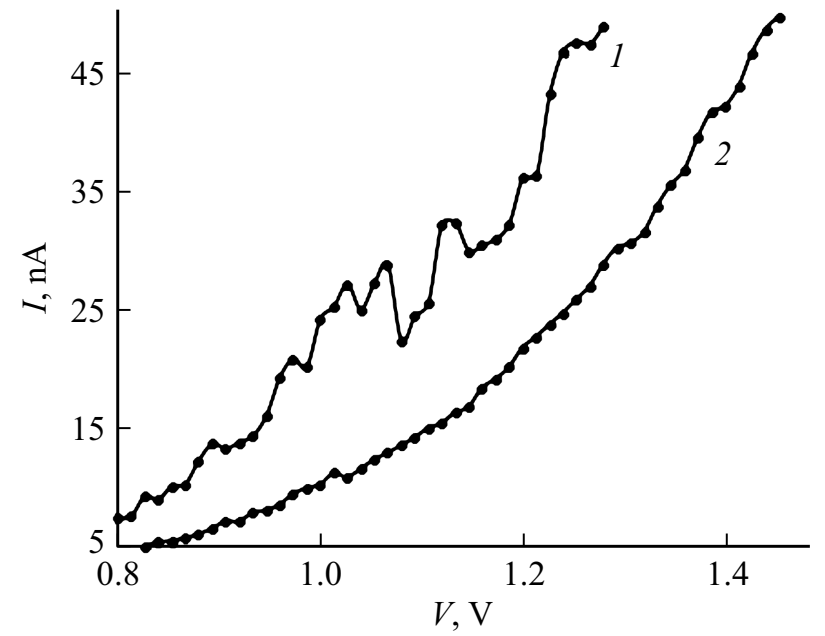

Рис. 4. Типичные BAX для образцов QD с особенностями (1) и без них (2).

ет вероятность фотолюминесценции. Поскольку с уменьшением температуры эффективная масса носителей заряда уменьшается, т.е. размерное квантование усиливается, относительная интенсивность фотолюминесценции уменьшается. Таким образом, можно предположить, что для квантовых точек с невысокими параметрами размерного квантования (QD-CdSe) действует экситонный механизм рекомбинации и фотолюминесценции, а для QD с высокими параметрами (QD-PbS) - конкуренция двух эффектов. При этом точкой максимума фотолюминесценции для QD-PbS является температура $70^{\circ} \mathrm{C}$ (рис. 3, кривая 1), а ее спад при комнатной температуре может быть весьма существенным (на рис. 3 - 2 раза).

Обнаруженные на ВAX особенности заключались в характерных нестабильности тока и провалах (рис. 4). На большом массиве измеренных ВАХ было установлено, что число образцов, имеющих особенности на $\mathrm{BAX}$, для примерно одинаковых размеров QD было: QD-CdSe $-\sim 15 \%$, QD-PbS $-\sim 40 \%$, QD-InSb $\sim 60 \%$, т.е. проявление особенности поведения ВАХ чаще для образцов по мере увеличения их параметров размерного квантования. Это можно объяснить в той же модели резонансного состояния электрона в глубокой протяженной потенциальной яме QD, - неравновесный электрон в процессе его транспорта в QD при определенной энергии (напряжении на образце) как бы „застревает“ в своем резонансном состоянии, а в других состояниях он просто выбрасывается из QD туннельной эмиссией, не создавая особенностей.

\section{4. Заключение}

На основе вариаций параметров синтеза квантовых точек и измерений на электронном и зондовом микроскопах установлено, что определяющей для структурнофизических свойств квантовых точек является скорость кристаллизации в процессе их коллоидного синтеза. При этом при медленном росте кристаллов гистограмма распределения по размерам имеет два максимума, что связано, вероятно, с термодинамической анизотропией кристаллизации. Исследованы форма и размеры коллоидных квантовых точек сульфида свинца в зависимости от времени синтеза при использовании несольватирующего растворителя прекурсора серы - уайт-спирита. Оценочно установлены формы и пределы размеров наночастиц совершенной кристаллической структуры. Обнаружены особенности в свойствах - аномальная температурная зависимость фотолюминесценции и нестабильный характер вольт-амперной характеристики, которые объяснены в модели размерного квантования энергии и импульса неравновесных электронов. Особенности проявляются тем сильнее, чем больше параметры размерного квантования, по возрастанию в следующей последовательности: $\mathrm{CdSe}, \mathrm{PbS}, \mathrm{InSb}$.

\section{Финансирование работы}

Работа выполнена при финансовой поддержке гранта 20-07-00307-a.

\section{Благодарности}

Авторы благодарны за помощь при измерениях А.М. Бурову (ЦКП „Симбиоз“ ИБФРМ РАН) и А.М. Захаревичу (лаборатория диагностики наноматериалов и структур СГУ).

\section{Конфликт интересов}

Авторы заявляют, что у них нет конфликта интересов.

\section{Список литературы}

[1] M. Alizadeh-Ghodsi, M. Pourhassan-Moghaddam, A. ZavariNematabad, B. Walker, N. Annabi, A. Akbarzadeh. Part. Part. Syst. Charact., 36, 1800302 (2019).

[2] С.Б. Бричкин, В.Ф. Разумов. Успехи химии, 85 (12), 1297 (2016).

[3] Y. Pu, F. Cai, D. Wang, J.X. Wang, J.F. Chen. Ind. Eng. Chem. Res., 57 (6), 1790 (2018). DOI: 10.1021/acs.iecr.7b04836

[4] D. Porotnikov, M. Zamkov. J. Phys. Chem. C, 124 (40), 21895 (2020).

[5] Z. Hens, J. De Roo. J. Amer. Chem. Soc., 142 (37), 15627 (2020).

[6] Y. Altintas, B. Liu, P. LudwigHernández-Martínez, N. Gheshlaghi, F. Shabani, M. Sharma, L. Wang, H. Sun, E. Mutlugun, H.V. Demir. Chem. Mater., 32 (18), 7874 (2020).

[7] D. Wang, F. Yin, Z. Du, D. Han, J. Tang. J. Mater. Chem. A, 7 (46), 26205 (2019).

[8] С.И.Борисенко. Физика полупроводниковых нанострукmyp: учебное пособие (Томск, изд-во Томск. политех. ун-та, 2010).

[9] Л.Б. Матюшкин, О.А. Александрова, А.И. Максимов, В.А. Мошников, С.Ф. Мусихин. Биотехносфера, 2, 28 (2013). 
[10] Д.В. Крыльский, Н.Д. Жуков. Письма ЖТФ, 45 (16), 10 (2019).

[11] С.В. Дежуров, А.Ю. Трифонов, М.В. Ловыгин, А.В. Рыбакова, Д.В. Крыльский. Рос. нанотехнол., 11 (5), 54 (2016).

[12] D. Wilkinson, Ch. Li. J. Phys. Chem. C, 114, 3329 (2010).

[13] F. Navarro-Pardo, H. Zhao, Z.M. Wang, F. Rosei. Acc. Chem. Res., 51, 609 (2018).

[14] Н.Д. Жуков, А.И. Михайлов, Д.С. Мосияш. ФТП, 53 (3), 340 (2019).

[15] Е.И. Гольдман, Ю.В. Гуляев, А.Г. Ждан, Г.В. Чучева. ФТП, 44 (8), 1050 (2010).

[16] A.I. Mikhailov, V.F. Kabanov, M.V. Gavrikov. Nanosystems: Physics, Chemistry, Matematics, 10 (6), 720 (2019).

[17] П.А. Алексеев, И.Е. Кононова, А.И. Максимов, Е.В. Мараева, В.А. Мошников. Диагностика материалов методами сканирующей зондовой микроскопии (СПб., Изд-во СПбГЭТУ „ЛЭТИ“, 2017).

[18] Н.Д. Жуков, Д.В. Крыльский, М.И. Шишкин, А.А. Хазанов. ФТП, $53(8), 1103$ (2019).

[19] P. Reiss, M. Protiere, L. Li. Small, 5 (2), 154 (2009).

[20] J. Tersoff. Phys. Rev. B, 40 (17), 11990 (1989).

[21] M.A. Hines, G.D. Scholes. Adv. Mater., 15 (21), 1844 (2003).

[22] Sijie Zhou, Zeke Liu, Yongjie Wang, Fan Yang, Mengfan Gu, Yalong $\mathrm{Xu}, \mathrm{Si}$ Chen, Xufeng Ling, Yannan Zhang, Fangchao Li, Jianyu Yuan, Wanli Ma. J. Mater. Chem. C, 7, 1575 (2019). DOI: 10.1039/c8tc05353g

[23] В.П. Драгунов, И.Г. Неизвестный, В.А. Гридчин. Основы наноэлектроники (М, Логос, 2006).

Редактор Г.А. Оганесян

\section{Properties of semiconductor colloidal quantum dots obtained under controlled synthesis conditions}

N.D. Zhukov ${ }^{1}$, T.D. Smirnova ${ }^{2}$, A.A. Khazanov' ${ }^{1}$, O.Yu. Tsvetkova ${ }^{1}$, S.N. Shtykov ${ }^{2}$

${ }^{1}$ RPP Volga Limited Liability Company, 410033 Saratov, Russia

${ }^{2}$ Saratov State University named after

N.G. Chernyshevsky,

410012 Saratov, Russia

Abstract The influence of the technological parameters of synthesis on the structural and physical properties of colloidal quantum dots based on lead sulfide, indium antimonide, and cadmium selenide has been investigated. The use of a solvent nonsolvating for the metalloid precursor allows one to obtain a slow, controlled process and trace in detail all stages of crystallization of nanoparticles - nucleation and growth, Ostwald ripening, uncontrolled growth. It is possible to obtain crystallites of perfect structure and size with minimal scatter - no more than $\pm 10 \%$. It was found that the maximum sizes of nanoparticles are limited by the thermodynamic growth conditions and have a value of about $5 \mathrm{~nm}$. In addition, quantum dots exhibit a number of specific properties - anomalous temperature dependence of photoluminescence and instability of the current-voltage characteristic, which are explained in the size quantization model of the energy and momentum of nonequilibrium electrons. For different variants of semiconductors, the features become more pronounced with an increase in the size quantization parameters. 